\title{
Evolutionary mechanism of water cycle and efficient utilization of water resources in the Haihe River Basin
}

Water cycle in the river basin constitutes the objective basis of water resources evolution. Whatever their manifestations, water resources problems can all attribute to the imbalances caused by the evolution of water cycle or its associated processes in the river basin. Various types of water activities for solution to water problems are in nature a regulation of "natural-social" dualistic water cycle or its associated processes in the river basin.

China is one of those countries which are faced with the most violent water cycle evolution and serious water problems with the Haihe River Basin as the most typical example. The Haihe River Basin has the least per-capita water resources among the ten first-grade river basins in China, but a dense population and developed economy. It now suffers from a prominent water resources decline, fierce competition between different water use sectors. In the view of the basic scientific and technological support required by water management and eco-environmental protection in the river basin, China in 2006 approved the National Basic Research Program of China entitled Evolutionary Mechanism of Water Cycle and Efficient Utilization of Water Resources in the Haihe River Basin, trying to build efficient utilization standards and patterns of urban-rural water resources on the basis of identifying the water cycle evolutionary mechanism in water-stressed river basins with intensive human activities.

Six major achievements have been made as follows through strenuous efforts of our project research team during the past five years. First, we have identified the evolutionary mechanism of water cycle and water resources in the river basin under a strong influence of human activities. Second, we have analyzed the evolutionary principle and driving mechanism of water chemistry and ecological process accompanied by the water cycle. Third, we have brought innovations of the integrated simulation and prediction technologies of "natural and social" dualistic water cycle and associated processes in the river basin. Fourth, we have discovered the evolutionary rules for water resources and eco-environment under a changing environment in the Haihe River Basin. Fifth, we have expounded the multi-scale urban and agricultural efficient water use mechanism and route in the Haihe River Basin. Sixth, we have built up the multiple integrated threshold regulation theory, threshold and pattern of water cycle in the Haihe River Basin. This project has been checked and accepted by the Ministry of Science and Technology of China in 2010. Many of its subjects have won the first-grade provincial-level awards and lots of its outcome has been applied effectively in national or local major water management practices, vigorously promoting China's basic and application research on water cycle simulation and regulation. Many of the achievements have been applied in national and local water management practices, vigorously promoting the basic and applied research on the simulation and regulation of water cycle in China.

This special topic includes the above research achievements as follows: A platform to comprehensively simulate dualistic water cycle and associated processes in the Haihe River Basin was developed. The evolutionary trend of water resources, water ecology and water environment were predicted. It holds that through implementing water-consumption control and management and south-to-north water transfer project, the water resources consumption, over-exploited underground water and water running into the seas can evolve well while there will be an increase in the water use for economic growth in the Haihe River Basin in 2030. Meanwhile, associated water environmental deterioration can be alleviated [1]; It was made clear that the evolutionary reasons and rules of temperature, precipitation and river systems in the Haihe River Basin in the past 10000 years, the energy transformation is the internal driver for water cycle flux evolution in the Haihe River Basin, and the temperature dominated and positively correlated to precipitation [2]; A water cycle multi-dimensional critical overall regulatory model system was established. The water cycle multi-dimensional critical overall regulatory threshold and total water quantity control objective were put forward in the Haihe River Basin [3]; A coupled hydrodynamic and water quality numerical model for river networks and lakes that applies to the river water environment system was developed [4]; The PAH pollution characteristics of the southern part of the Haihe River system were investigated, and a risk quotient (RQ) was used to assess the ecological risk of PAHs. The total PAH concentration ranged from 258.77 to 11296.66 ng/g dry weight [5]; Con- 
centrations of polycyclic aromatic hydrocarbons (PAHs) adsorbed on street dust in different size ranged and their correlation with specific surface area and total organic carbon (TOC) were investigated [6]; Using a summer maize field in North China as an example, the variations in leaf and canopy photosynthesis and transpiration (or evapo-transpiration) were analyzed [7]; The principal component analysis (PCA) and geographically weighted regression (GWR) were combined to estimate the spatial distribution of water requirement of the winter wheat in North China [8].

In order to share the above-mentioned academic achievements, Chinese Science Bulletin has set up a special topic to present you with representative research outcome. By so doing, we sincerely hope our counterparts home and abroad to oblige us with your valuable comments and opinions.

1 Wang H, Jia Y W, Yang G Y, et al. Integrated simulation of the dualistic water cycle and its associated processes in the Haihe River Basin. Chin Sci Bull, 2013, 58: 3297-3311

2 Liu J H, Xu H, Qin D Y, et al. Water cycle evolution in the Haihe River Basin in the past 10000 years. Chin Sci Bull, 2013, 58: 3312-3319

3 Gan H, Wang L, Cao Y B, et al. Multi-dimensional overall regulatory modes and threshold values for water cycle of the Haihe River Basin. Chin Sci Bull, 2013, 58: 3320-3339

4 Wang J H, Xiao W H, Wang H, et al. Integrated simulation and assessment of water quantity and quality for a river under changing environmental conditions. Chin Sci Bull, 2013, 58: 3340-3347

5 Liu F, Liu J L, Chen Q Y, et al. Pollution characteristics and ecological risk of polycyclic aromatic hydrocarbons (PAHs) in surface sediments of the southern part of the Haihe River system in China. Chin Sci Bull, 2013, 58: 3348-3356

6 Li Y X, Xiang L, Tian P, et al. Desorption characteristics of total phosphorus and heavy metals from impervious urban surface sediments. Chin Sci Bull, 2013, 58: 3357-3360

7 Zhang B Z, Liu Y, Xu D, et al. Water-carbon coupling modeling of summer maize at the leaf and canopy scales. Chin Sci Bull, 2013, 58: 3361-3370

8 Wang J L, Kang S Z, Sun J S, et al. Estimation of crop water requirement based on principal component analysis and geographically weighted regression. Chin Sci Bull, 2013, 58: 3371-3379

Guest Editor: WANG Hao

State Key Laboratory of Simulation and Regulation of Water Cycle in River Basin, China Institute of Water Resources and Hydropower Research, Beijing 100038, China

Open Access This article is distributed under the terms of the Creative Commons Attribution License which permits any use, distribution, and reproduction in any medium, provided the original author(s) and source are credited. 